\title{
Estimating Motion in Ultrasound Images of the Small Bowel: Optical Flow without Image Structure
}

\author{
David H. Cooper, Bo René Madsen, and Jim Graham \\ University of Manchester, Imaging Science and Biomedical Engineering \\ Stopford Building, Oxford Road, Manchester M13 9PT, UK \\ Jim.Graham@man.ac.uk
}

\begin{abstract}
Measurement of motion in transabdominal ultrasound sequences of the small bowel is hampered by the unstructured, textured appearance of the images. We investigate a method of measuring Optical Flow in the sequences, based on an analysis of the spatio-temporal frequency spectrum. The method requires no unrealistic assumptions about the distribution of energy in the frequency spectrum and extends readily to deal with the aperture problem. Evaluation on synthetic image sequences indicates it has advantages in accuracy and precision of velocity estimates. Qualitative evaluation on real ultrasound sequences indicate encouraging performance, provided occurrence of the aperture problem is identified and appropriate processing applied.
\end{abstract}

\section{Introduction}

This study forms part of a project to identify methods of measuring peristaltic motion in transabdominal ultrasound sequences of the bowel. Accurate motion measurements could make it possible to use transabdominal ultrasound, rather than barium radiology with its high radiation exposures, as the initial radiological investigation for patients with suspected or recurrent bowel disease. The appearance of inflammatory bowel disease in ultrasound sequences involves thickening of the bowel wall and reduced or absent peristalsis (motion of the bowel). Interpretation of the images can be difficult, and there are many equivocal cases. The ultrasound image appearance is mainly amorphous and textured with little or no recognisable structure. When bowel structure is apparent it shows highly variable, constantly changing shape. Figure 3(a) shows part of a typical frame; the marked points refer to analysis described in section 3 . The peristaltic movement of the bowel appears as slow movements of textured regions.

The literature on image motion measurement is vast, and it cannot be reviewed other than superficially here. For this application we discount methods that involve tracking of identifiable structures (e.g. [1,2]), as ultrasound images often give rise to spurious features (e.g. [3]). Studies of motion in ultrasound images have largely concerned echocardiogram sequences, where there is clear image structure (albeit noisy). The absence of recognisable features that can be modelled suggests that optical flow - detecting the changes in the brightness pattern - may be a more useful approach to the bowel ultrasound images. Optical flow methods may be thought of in two types: gradient-based methods (mainly improvements or variations on the 
original proposal by Horn and Schunk [4, 5], for example [6]), and methods that make use of the frequency-domain spectrum. The textured ("noisy) appearance of the images renders gradient-based (differentiating) methods unpromising. Baraldi [7] conducted an evaluation of several gradient-based methods on different synthesized sequences simulating ultrasound scatterers and detectors. There was considerable variability in results depending on the nature of the simulation, and considerable spatial smoothing was required in all cases. Similarly Mailloux [3] rejected several gradient based methods on echocardiogram sequences, again noting that to obtain useful performance significant image smoothing was required.

Frequency domain methods make velocity estimates by integrating over some neighbourhood in space and time, and provide a potentially useful avenue of investigation. The principles underlying frequency domain methods of optical flow are straightforward.

The energy in the Fourier spectrum of a 2D signal, $f(x, y)$, moving with constant translational velocity $(u, v)$, resides entirely in a plane in frequency

$\left(\omega_{x}, \omega_{y}, \omega_{t}\right)$ space, the orientation of which is determined by the velocity. Estimating the velocity is equivalent to estimating the orientation of this plane [8].

The most well known approaches to doing this are those of Bigün et al. [9], Fleet et al. [10], and Heeger [8]. Bigün et al formulate the problem as an eigenvalue problem but their method involves calculating derivatives in space-time, and is therefore not suitable for our application. An important issue in optical flow is dealing with the aperture problem. It may be possible to make reliable velocity estimates only in the direction normal to the local image gradient (we might speak of "normal flow" rather than "full flow"). Fleet et al [10] suggest that isocontours of the local phase (rather than amplitude) provide a more robust indicator in these circumstances. Liu et al [11] have found Fleet's method to be very noise sensitive. Our own investigations [12] support this and we find the same to be true of Bigün's method.

Heeger's method estimates the velocity by sampling the local power spectrum using Gabor filters tuned to particular spatio-temporal frequencies. The plane orientation, and hence the velocity, can be estimated by minimising the difference between predicted and observed outputs of the filters. While Heeger's method appears to fulfil our requirements, in application to synthetic images we have found the errors in velocity estimation to be larger than we would like (see figure 2 ). We believe this is due to two problems. Firstly Heeger's method makes the assumption that the energy of the spectrum is uniformly distributed within the plane. This condition is difficult to meet even with realistic synthetic images, and is highly unlikely to be satisfied by real data. Secondly, the method is likely to give unreliable results in the presence of the aperture problem. Heeger proposed that the error surface in $(u, v)$ space is ridgeshaped, rather than a minimum, when the aperture problem applies. Barron et al. [13] suggested a heuristic method of identifying this condition, though in our experience this does not always work well for real image data [14].

It is easily shown [12] that when the aperture problem arises, the energy is concentrated into a line in frequency space. Measuring the normal flow component amounts to calculating the orientation of the line. 


\section{Estimating the velocity}

Here we describe a method of estimating the velocity by direct search in frequency space. At each point $\mathbf{x}(x, y, t)$ for which we wish to calculate a velocity we seek a maximum in the power in a plane ("full-flow") or a line ("normal-flow") in its power spectrum defined by a given $\left(\theta_{m}, \varphi_{m}\right)$ over all $(\theta, \varphi)$ in a hemisphere. No assumptions are required concerning the distribution of energy in the line or plane. However, we need to retain the assumption that the motion consists of uniform translation. In any realistic situation, this assumption will not generally hold over extended regions of space and time. Ideally we would make velocity estimates at individual points in space-time, but we need data over some spatial and temporal extent to be able to calculate the FTs. We compromise by calculating the velocity for a volume of spacetime centred on the point $\mathbf{x}$ at which the velocity is required. For our experiments (section 3) we use cuboids of $13^{3}$ points for full-flow analysis and $7^{2} \times 13$ for the normal-flow, (reflecting the one-dimensional spatial extent of the sample). These values are, of course somewhat arbitrary, and were chosen to be compatible with the support region for Heeger's Gabor filters.

To conduct our search we need a method of calculating the power in a given plane or line and an optimisation strategy.

\subsection{Calculating the power}

We have two choices for calculating the power in a plane or line: by calculating the FT and working in frequency space or directly from the spatio-temporal data. In either case we make use of some standard results concerning the Fourier transform [15]. The rotation theorem allows us to calculate the power in any plane or line by rotating the volume of data by $(-\theta,-\varphi)$ and calculating the power in the plane $\omega_{t}=0$ or the line $\left(\omega_{x}=0, \omega_{y}=0\right)$. For any rotated volume of space, we can calculate the FT and sum the power values over the plane $\omega_{t}=0$ or along the $\omega_{x}$ axis. To calculate the power using the space-time domain data we use two further theorems. Consider the case of full-flow analysis. To obtain the integrated power in the plane $\omega_{t}=0$ we need to multiply the spectrum by a filter, which has the value 1 everywhere in the plane and 0 elsewhere. This is just a delta function $\delta\left(\omega_{t}\right)$, the FT of which is a constant. By virtue of the convolution theorem we can achieve this in the spatio-temporal domain by convolution with a constant function in the time axis only. For a truncated volume of data the power in the plane $\omega_{t}=0$ can be simply estimated by summing in time the data values at each $(x, y)$ position, then summing the squares of the result over the plane (Rayleigh's theorem).

Either of these approaches allows us to measure the power in any chosen plane of frequency space, without making any assumptions about the distribution of power within that plane. A similar approach can also be taken to measure the power in a line for normal-flow analysis. Which approach to take is entirely a matter of efficiency. In our experiments we used the space-time convolution for full-flow and the FT for normal-flow. 


\subsection{Estimating the orientation}

We evaluate two methods of locating the line or plane.

Multi-scale (coarse-to-fine) search of $(\theta, \varphi)$ space: Each scale is selected by gaussian convolution of the rotated volume. At each scale an exhaustive search is conducted over a $3 \times 3$ neighbourhood of the $(\theta, \varphi)$ map centred at the value of $(\theta, \varphi)$ yielding the maximum power at the previous scale. In our experiments we have used 10 scales.

Principal Component Analysis (PCA) of the spectrum: This provides a means of distinguishing between full-flow and normal-flow conditions. If the spectrum is predominantly linear, one of the eigenvalues will be much greater than the other two. If it is planar, there should be two similar eigenvalues, much higher than the third. The orientation is specified by the eigenvector whose eigenvalue is either the highest (in the case of a line) or lowest (in the case of a plane).

This initial estimate of plane or line orientation by either method can be refined by local search at the finest scale. In the experiments described below, we use the fmins function in the MATLAB optimisation toolbox, which is an implementation of the Nelder-Mead (simplex) method [16].

\section{Experiments}

\subsection{Quantitative evaluation: synthetic data}

Two types of synthetic images were used to conduct quantitative evaluations.

Random texture image: an image of random grey levels, providing an approximation to a texture of uniform energy from which full-flow analysis should be possible.

Random lines image: a random 1-D pattern is projected to form lines at a given orientation. Full-flow analysis is impossible, but we should be able to calculate normal-flow, the direction of the flow vector being normal to the lines. Although these images show a preferred direction, no image gradient is present.

For each of these image types a synthetic sequence was generated by simulating motion of the image in various directions at different speeds.

Figure 1 is an illustration of the $(\theta, \varphi)$ maps that result from application of the fullflow and normal-flow analyses to the random texture and random line images. Where the method seeks a plane in the full-flow case or a line in the normal-flow case, a peak is obtained in the map. When the method is applied in the "wrong" condition (seeking a plane in the normal-flow case, for instance) the result is a sinusoidal ridge.

Figure 2 shows a comparison between full-flow estimation in a synthetic sequence $\left(128^{2}\right.$ pixels), using our implementation of Heeger's method and the full-flow method using coarse-to-fine search. The velocity $(u, v)$ in this case is $(0.3,0.7)$ pixels per frame. The absolute value in the mean error (not easily discernible in the figure) is 0.26 pixels per frame for Heeger's method and 0.03 pixels per frame for our direct search method. The improvement in the dispersion of values is obvious, reduced from a standard deviation of 0.15 to 0.02 (on absolute velocity). 

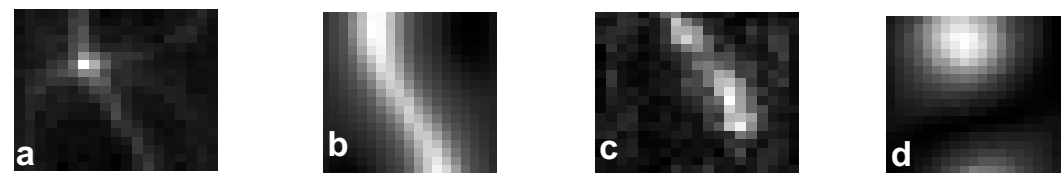

Fig. 1. Examples of $(\theta, \varphi)$ maps for synthetic sequences with velocities of $(0.5,0.5)$. Full-flow analysis applied to the random texture image (a) and the random line image (b) (lines at $30^{\circ}$ ). Normal-flow analysis applied to the random texture image (c) and the random lines image (d). Where analysis and flow conditions match there is a peak, where they do not there is a ridge
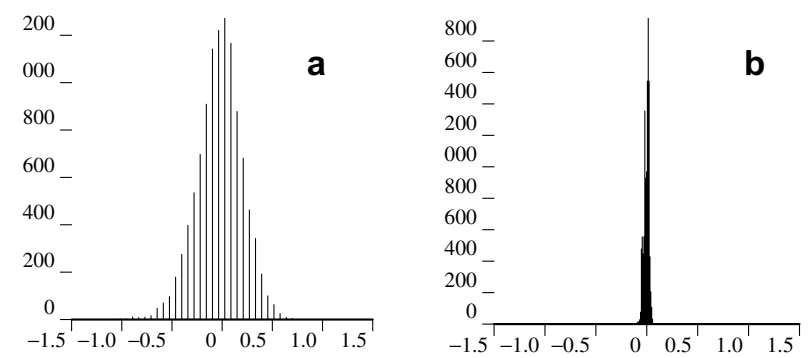

Fig. 2. Distribution of error in the u-component of a random-texture sequence, measured using Heeger's method (a) and direct search (b). The mean error is reduced (see text) and the dispersion is greatly reduced. Similar results are obtained for the v-component

Quantitative results on flow measurement on synthetic sequences are summarised in Table 1. Each result is derived from a number of synthetic uniform motion sequences of a square of $31 \times 31$ pixels ( 961 velocity estimates) with combinations of $(u, v)$, each in the range $0 . .1$ (a requirement of both Heeger's method and ours). Twenty translation sequences were used for full-flow experiments, eighteen for normal-flow (6 different velocities and 3 different orientations of the line pattern). The mean and s.d. for both components of the velocity: $m_{u}, m_{v}, \sigma_{u}, \sigma_{v}$ are shown. The error for each sequence is the difference between the true velocity and the mean $\left(u-m_{u}, v-m_{v}\right)$. Four variants of our search strategy are compared: coarse-to fine search or PCA providing initial estimates, with and without local search.

The errors in velocity estimates and the dispersion of estimates are small for all four search algorithms, both for full-flow and normal-flow analyses. Direct assessment of the line or plane by PCA alone is the least successful of the methods. However, using PCA as a starting estimate, subsequently refined by local search produces results hardly distinguishable from coarse-to-fine search. Local search following coarse-to-fine search produces very little improvement in accuracy or precision of velocity estimates. The difference between PCA with local search and coarse-to-fine search is more marked in the case of normal-flow analysis, where the coarse-to-fine search achieves better estimates. 
Table 1. Summary of velocity estimation errors for the synthetic sequences (a) Full-flow estimates on the random-texture sequence. (b) Normal-flow estimates on the random-lines sequence. The search strategies are PCA or coarse-to-fine (C-F) on its own or followed by local search (+S). $\left|e_{u}\right|$ and $\left|e_{v}\right|$ are the average values of the absolute errors in each sequence. $\sigma_{u}$ and $\sigma_{v}$ are the average values of the standard deviations of velocity measurements.

\begin{tabular}{|l|l|l|l|l|}
\hline Method & PCA & $\begin{array}{l}\text { PCA } \\
+\mathbf{S}\end{array}$ & $\mathbf{C - F}$ & $\begin{array}{l}\text { C-F } \\
+\mathbf{S}\end{array}$ \\
\hline$\left|\boldsymbol{e}_{\boldsymbol{u}}\right|$ & 0.063 & 0.017 & 0.019 & 0.019 \\
\hline$\left|\boldsymbol{e}_{\boldsymbol{v}}\right|$ & 0.066 & 0.02 & 0.022 & 0.019 \\
\hline $\boldsymbol{\sigma}_{\boldsymbol{u}}$ & 0.025 & 0.027 & 0.028 & 0.027 \\
\hline $\boldsymbol{\sigma}_{\boldsymbol{v}}$ & 0.024 & 0.028 & 0.028 & 0.026 \\
\hline
\end{tabular}

a

\begin{tabular}{|l|l|l|l|}
\hline PCA & $\begin{array}{l}\text { PCA } \\
+\mathbf{S}\end{array}$ & $\mathbf{C}-\mathbf{F}$ & $\begin{array}{l}\mathbf{C}-\mathbf{F} \\
+\mathbf{S}\end{array}$ \\
\hline 0.014 & 0.011 & 0.006 & 0.005 \\
\hline 0.043 & 0.03 & 0.013 & 0.013 \\
\hline 0.007 & 0.02 & 0.028 & 0.028 \\
\hline 0.016 & 0.028 & 0.06 & 0.06 \\
\hline
\end{tabular}

b

\subsection{Qualitative evaluation: medical ultrasound sequences}
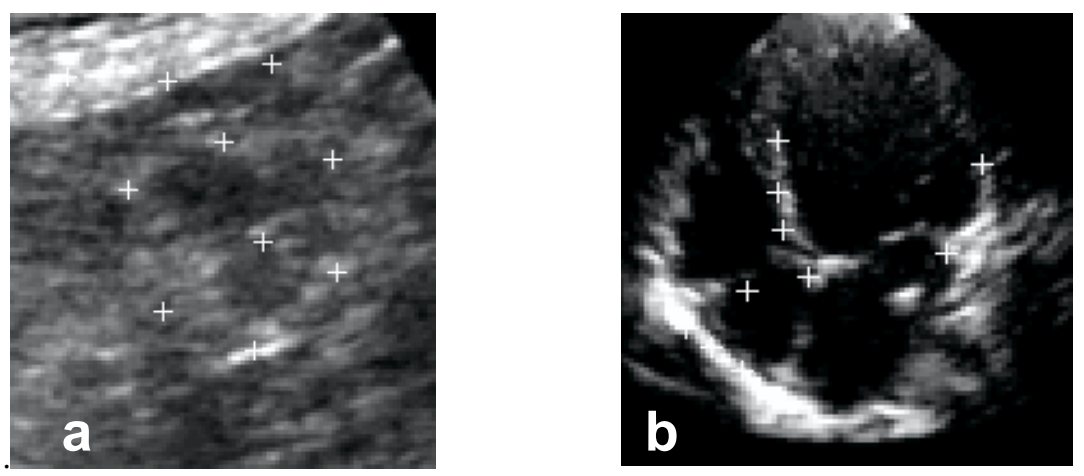

Fig. 3. (a) Single frame from an ultrasound sequence of the small bowel. The marked points have been positioned for tracking (see text). Very little structure is visible. Some of the marked points form a circle corresponding to the circumference of a cross-section of bowel which is just discernible in the centre of the frame.

(b) A frame from an echocardiogram sequence with points marked for tracking.

Evaluation on real data is of necessity a more subjective exercise than on synthetic data, due to the absence of ground truth. Even subjective assessment is difficult in the case of the bowel images because of the lack of consistent structure. To assist in the qualitative evaluation we use, in addition to the bowel ultrasound sequences, echocardiogram sequences of the left ventricle in which anatomical structure is much clearer, making visual assessment of motion easier. One of our original design criteria was to find a method of optical flow estimation that would give us results in the absence of structure. However, the presence of structure should not be a disadvantage to our direct search method, as we need no assumptions about the distribution of power in the line or plane. Figure 3 shows frames from a bowel sequence (a) and an 
echocardiogram sequence (b). In both cases certain points have been marked, and we use these points to evaluate the flow methods by tracking. On each frame the velocity is calculated at each point and its position on the next frame estimated. Good estimates of velocity will result in the points "tracking" the underlying structure. (Recall that we are not "tracking" by structural correspondence.) The results of this evaluation cannot be visualised on paper, as it is necessary to watch the sequences unfold. Here we describe the subjective assessment of tracking using full-flow analysis and normal-flow analysis, both by coarse-to-fine search.

While tracking in the bowel sequence appears usually (but not always) convincing using full-flow analysis, the results with the echocardiogram sequence are poor; tracking in this case is extremely unstable. This is unsurprising, since the pronounced structure results in conditions where the aperture problem will arise. Improved tracking results are obtained in both sequences by using normal-flow analysis. In the echocardiogram sequences the normal-flow tracking is extremely stable.

\section{Conclusions}

The Frequency-domain approach to optical flow is not widely used. In their evaluation of optical flow methods, Barron et al. [13] found that Heeger's method performed as well as other methods on synthetic image sequences, though they had difficulty in applying it to realistic images due to its failure on occasions to converge. The more recent evaluation by McCane et al [17] did not evaluate any frequencydomain methods, nor did they use any sequences that closely synthesized the properties of the bowel ultrasound images. Our use of the frequency-space approach arises from a rejection of other methods due to experience of others, argument from principle and some objective evaluation [12]. The wide variety of approaches in the literature means that we cannot claim to have exhaustively and rigorously considered all possible alternatives. For example Black and Anandan [18] show useful results of robust patch tracking on synthetic images similar to those that we have used, although the real sequences they used are not comparable to the bowel sequences.

We have developed our frequency-space method of measuring optical flow method in response to the requirements of a particular, challenging application. We know of no previous study that has attempted to measure motion in transabdominal ultrasound sequences. This method is applicable in other areas of application in which a frequency-domain approach to optical flow is appropriate. It demonstrates greater accuracy and precision than Heeger's method (in our hands) when applied to synthetic motion sequences. It offers the additional benefits of being more generic in requiring no restrictive assumptions about the power distribution in the image spectrum, of dealing naturally with the presence or absence of the aperture problem and of being conceptually straightforward. The accuracy and precision on synthetic sequences and the stable results on real sequences provide encouragement that realistic motion estimates are possible in real medical ultrasound investigations. A key issue in these applications is the ability to recognize and deal naturally with the aperture problem. 


\section{References}

1. S. Ullman, The interpretation of visual motion. Cambridge, MA: MIT Press, 1979.

2. A. Singh and P. Allen, "Image flow computation: an estimation-theoretic framework and a unified perspective", CVGIP - Image Understanding, 56, 152177, 1992.

3. G. Mailloux, A. Bleau, M. Bertrand and R. Peticlerc, "Computer analysis of heart motion from two-dimensional echocardiograms", IEEE Transactions on Biomedical Engineering, 34, 356-364, 1987.

4. B. Horn and B. Schunk, "Determining optical flow", Artificial Intelligence, 17, 185-203, 1981.

5. B. Horn and B. Schunk, "Determining optical flow - a retrospective", Artificial Intelligence, 59, 81-87, 1993.

6. A. Verri, F. Girosi and V. Torre, "Differential techniques for optical flow", Journal of the Optical Society of America. A, 912-922, 1990.

7. P. Baraldi, A. Sarti, et al., "Evaluation of differential optical flow techniques on synthesised echo images", IEEE Transactions on Biomedical Engineering, 43, 259-272, 1996.

8. D. J. Heeger, "Optical flow using spatio-temporal filters", Int.J. Computer Vision, 1, 279-302, 1988.

9. J. Bigün, G. H. Granlund and J. Wiklund, "Multidimensional orientation estimation with application to texture analysis and optical flow", IEEE Trans. PAMI, 13, 775-790, 1991.

10. D. J. Fleet and A. D. Jepson, "Computation of component image velocity from local phase information", Int. J. Computer Vision, 5, 77-104, 1990.

11. H. Liu, T.-H. Hong, M. Herman and R. Chellapa, "Accuracy vs. efficiency tradeoffs in optical flow algorithms", proc. European Conference on Computer Vision, Cambridge, U.K., 1996, pp. 174-183.

12. D. H. Cooper, "Methods for Motion Estimation in Medical Ultrasound Imaging", University of Manchester, Ph.D. Thesis, 2000.

13. J. Barron, D. Fleet, J. and S. S. Beauchemin, "Performance of Optical Flow Techniques", Int. J. Comput. Vis., 12, 43-47, 1994.

14. D. H. Cooper and J. Graham, "Estimating motion in noisy, textured images: Optical flow in medical ultrasound", proc. British Machine Vision Conference, Edinburgh, Scotland, 1996, pp. 585-594.

15. R. N. Bracewell, The Fourier Transform and its Applications, 2 ed: McGrawHill, 1986.

16. W. H. Press, S. A. Teukolsky, W. T. Vettering and B. P. Flannery, Numerical Recipes in C: The Art of Scientific Computing, 2 ed: Cambridge University Press, 1992.

17. B. McCane, K. Novins, D. Crannitch and B. Galvin, "On benchmarking optical flow", Comput. Vis. Image Underst., 84, 126-143, 2001.

18. M. J. Black and P. Anandan, "The robust estimation of multiple motions: Parametric and piecewise-smooth flow fields", Comput. Vis. Image Underst., 63, 75-104, 1996. 